\title{
Immune checkpoint inhibitors therapy in older patients $(\geq 70$ years) with metastatic melanoma: a multicentre study
}

\author{
Bożena Cybulska-Stopa ${ }^{1}$, Iwona Ługowska ${ }^{2,3}$, Paulina Jagodzińska-Mucha², Hanna Koseła-Paterczyk², \\ Katarzyna Kozak ${ }^{2}$, Anna Klimczak², Tomasz Świtaj², Marek Ziobro' ${ }^{1}$, Agnieszka Roman', Marcin Rajczykowski ${ }^{4}$, \\ Rafał Suwiński ${ }^{4}$, Maciej Niemiec ${ }^{1}$, Tomasz Zemełka ${ }^{1}$, Sławomir Falkowski², Piotr Rutkowski ${ }^{2}$ \\ ${ }^{1}$ Clinical Oncology Clinic, Maria Sklodowska-Curie Institute - Oncology Center, Cracow Branch, Poland \\ 2Department of Soft Tissue/Bone Sarcoma and Melanoma, Maria Sklodowska-Curie Institute - Oncology Center, Warsaw, Poland \\ ${ }^{3}$ Early Phase Clinial Trials Unit, Maria Sklodowska-Curie Institute - Oncology Center, Warsaw, Poland \\ ${ }^{4}$ Radiotherapy and Chemotherapy Clinic and Teachng Hospital, Maria Sklodowska-Curie Institute - Oncology Center, Gliwice Branch, \\ Poland \\ Adv Dermatol Allergol 2019; XXXVI (5): 566-571 \\ DOI: https://doi.org/10.5114/ada.2018.79940
}

\begin{abstract}
Introduction: The use of immunotherapy in older patients remains challenging due to very few data on the efficacy and safety of treatment in this group.

Aim: To analyse the efficacy and safety of immunotherapy with checkpoint inhibitors in older patients ( $\geq 70$ years) with metastatic melanoma.

Material and methods: In the Maria Skłodowska-Curie Institute - Oncology Centre, between 2011 and 2017, 318 non-resectable or metastatic melanoma patients were treated with immune checkpoint inhibitors: anti-CTLA-4 or/ and anti-PD-1. Eighty-two patients were $\geq 70$ years (median age: 76 years; range: $70-90$ years). Among this group $10 \%$ of patients had brain metastases, $24 \%$ of patients had BRAF mutant melanoma, and co-morbidities were present in $86 \%$ of patients (mainly hypertension, cardiovascular diseases and/or diabetes).

Results: Median PFS and OS were similar in patients $<70$ years and $\geq 70$ years. In the group of patients $\geq 70$ years old, the 2-year OS rate (from the start of immunotherapy) was $27 \%$, and in patients aged $<70$ it was $28 \%$ ( $p=N S$ ). Two-year progression-free survival was $13.7 \%$ in the group of patients $\geq 70$ years old and in patients aged $<70$ it was $13 \%(p=N S)$. Patients $\geq 70$ years of age were significantly less likely to have a BRAF mutation $(p=0.020)$. The presence of co-morbidities was not associated with an increased risk of immunotherapy $(p=0.790)$.

Conclusions: The survival and toxicity profile in the older patients treated with immune checkpoint inhibitors are similar to younger patients. Therefore, the age as a clinical factor should not exclude this population from the most effective therapy used nowadays in melanoma treatment.
\end{abstract}

Key words: melanoma, immunotherapy, older patients, anti-CTLA-4, checkpoint inhibitors, anti-PD-1, anti-PD-L1.

\section{Introduction}

The incidence of melanomas has increased significantly in recent years and reached over 87000 new melanoma cases and near 10000 deaths in the USA in 2017 [1]. Also the probability of developing melanoma increases with age and is about $0.5 \%$ at the age of 0-59 and $2.5 \%$ (men) and $1 \%$ (women) at the age of $\geq 70$ years [1]. The median age at initial melanoma diagnosis is 63 and the highest percentage of melanoma-related deaths occur in patients aged 75-84 [2]. For these reasons, the treatment of older patients with melanoma is becoming an increasing problem.

Melanoma is considered one of the immunogenic - if not the most immunogenic - malignancies which leads melanomas to respond to immunotherapy [3]. Recently, new developments in immunotherapy have revolutionized this treatment modality, namely immune checkpoint inhibitors. Immune checkpoint inhibitors target the "brakes" on the immune system, with the goal of inducing immune cell proliferation and activation against

Address for correspondence: Bożena Cybulska-Stopa, Clinical Oncology Clinic, Maria Skłodowska-Curie Institute - Oncology Centre, Cracow Branch, 11 Garncarska St, 31-209 Krakow, Poland, phone: +48 60794 17 15, e-mail: bcybulskastopa@vp.pl Received: 11.04.2018, accepted: 25.06.2018. 
cancer cells [4]. The best characterized and most therapeutically relevant immune checkpoint inhibitors are antibodies against cytotoxic T-lymphocyte-associated antigen 4 (CTLA-4) and antibodies against the programmed cell death protein-1 (PD-1) or its ligand (PD-L1). Immune checkpoint inhibitors have shown significant efficacy in the treatment of melanoma patients, which has been confirmed in many clinical trials [5-9].

Older patients with metastatic melanoma have different disease characteristics and they also can have a poorer prognosis than younger patients. One of the factors that may contribute to poor prognosis in older patients with metastatic melanoma is the weakening of the immune system with age [10]. This process is called immunosenescence [11]. Aging-associated thymic involution results in decreased numbers of naïve T cell relative to the memory T cells [10]. In older patients T cell functionality is decreased due to loss of co-stimulatory molecules such as CD28, T cell exhaustion, and reduction in pro-inflammatory cytokine secretion [12]. Longitudinal studies in the aging population have identified immune risk phenotypes that revealed low CD4 counts and reversed CD4 : CD8 ratio that correlated with a poor prognosis for patients with cancer [10]. Additionally, older patients often have many comorbidities and use a lot of concomitant medications. The use of immunotherapy in older patients remains challenging due to very few data on the efficacy and safety of treatment in this group. In some cases, unpredictable toxicity including interactions with other medications may occur and can lead to immunotherapy termination.

\section{Aim}

The aim of this study is to analyse the efficacy and safety of immune checkpoint inhibitors therapy in older patients ( $\geq 70$ years) with unresectable or metastatic melanoma.

\section{Material and methods}

\section{Patients}

In this multicentre, retrospective study, we evaluated the safety and efficacy of immune checkpoint inhibitors. We performed analysis of all patients with non-operative or metastatic melanoma who were treated with immune checkpoint inhibitors in one of three branches of the Maria Skłodowska-Curie Institute - Oncology Centre (Warsaw, Cracow, Gliwice) in 2011-2017. We analysed only patients with histologically confirmed diagnosis of skin or mucosal melanoma (patients with ocular melanoma were not included in the analysis). The clinical factors, concomitant diseases and medications, as well as applied treatment and survival outcomes were collected from patients' medical records.
All patients were treated with immune checkpoint inhibitors until disease progression or unacceptable toxicity. As the first-line immunotherapy we used anti-PD-1: nivolumab ( $3 \mathrm{mg} / \mathrm{kg}$ every 2 weeks), pembrolizumab ( $2 \mathrm{mg} / \mathrm{kg}$ every 3 weeks), or anti-CTLA-4: ipilimumab ( $3 \mathrm{mg} / \mathrm{kg}$ every 3 weeks up to four doses). As the second-line treatment of immunotherapy, ipilimumab or nivolumab/pembrolizumab (scheme as above) were used if not applied in the first-line treatment. The treatment scheme is presented in Figure 1.

Patients were excluded from the immunotherapy on the basis of contraindications to immune checkpoint inhibitors described in the Medicinal Product Characteristics (e.g. presence of symptomatic brain metastases; other concurrent systemic anticancer treatments for melanoma or presence of concurrent malignant disease with the exception of adequately treated basal or squamous cell skin cancer, superficial bladder cancer, or carcinoma in situ of the cervix).

Tumour response was assessed according to the Response Evaluation Criteria in Solid Tumours (RECIST), version 1.1 [13], 12 weeks after the start of treatment immunotherapy, and then every 12 weeks until disease progression or treatment discontinuation. Assessments for survival were performed every 3 months. Safety evaluations were performed for patients who received at least one dose of the immunotherapy, and adverse events were graded according to the National Cancer Institute Common Terminology Criteria for Adverse Events, version 4.0 [14].

\section{Statistical analysis}

All statistical analyses were performed using Stata Statistical Software: Release 13. The survival was assessed with respect to the following variables: age at the start of therapy: $<70$ or $\geq 70$ years. In most studies, the cut-off for age was 65 years [15] but in one of the largest analyses of immunotherapy in the elderly (the Italian

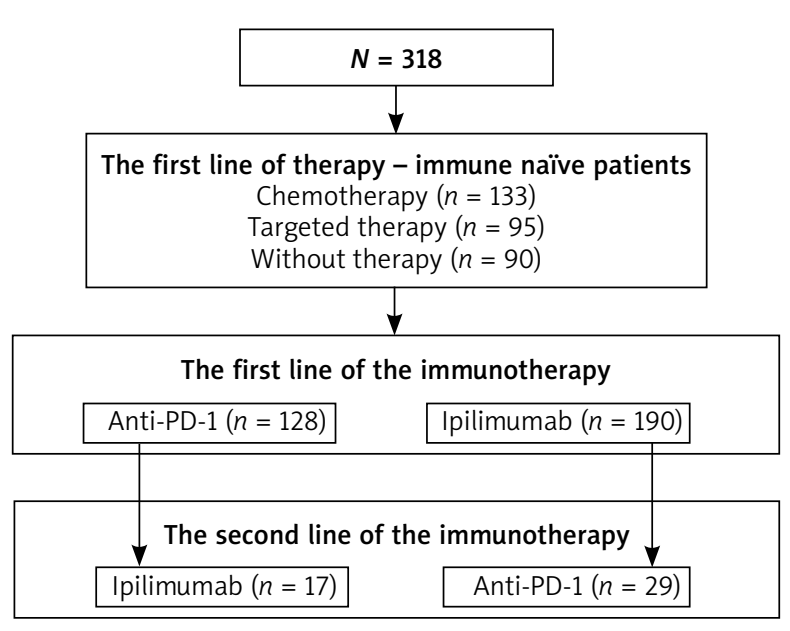

Figure 1. The treatment scheme. The initiation of immunotherapy was defined as the first line of immunotherapy 


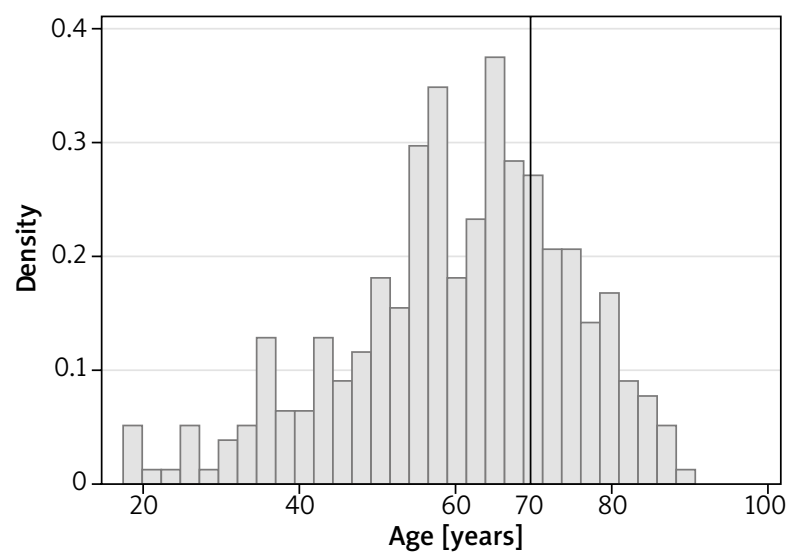

Figure 2. The histogram of the population's age

Table 1. Baseline patient characteristics

\begin{tabular}{lccc}
\hline Parameter & $\begin{array}{c}<70 \\
\text { years old }\end{array}$ & $\begin{array}{c}\mathbf{7} 0 \text { years } \\
\text { old }\end{array}$ & P-value \\
\hline $\begin{array}{l}\text { Total number of patients } \\
(N=318)\end{array}$ & 236 & 82 & \\
\hline $\begin{array}{l}\text { Sex, } n(\%): \\
\text { Male }\end{array}$ & $137(58)$ & $51(62)$ & \\
\hline Female & $99(42)$ & $31(38)$ & \\
\hline BRAF status, $n$ (\%): & & & 0.011 \\
\hline Positive & $92(39)$ & $19(23)$ & \\
\hline Negative & $144(61)$ & $63(77)$ & \\
\hline History of brain metastases, & & & 0.185 \\
$n(\%):$ & $37(16)$ & $8(10)$ & \\
\hline Yes & $199(84)$ & $74(90)$ & \\
\hline No & & & 0.001 \\
\hline Comorbidities, $n$ (\%): & $28(12)$ & $69(84)$ & \\
\hline Yes & $208(88)$ & $13(16)$ & \\
\hline No & & & 0.462 \\
\hline Lactate dehydrogenase, & $103(44)$ & $32(39)$ & \\
$n(\%):$ & $133(66)$ & $50(61)$ & \\
\hline Elevated & & & \\
\hline Normal & & & \\
\hline
\end{tabular}

study), cut-off for age was 70 years [16]. Median age of advanced melanoma patients is $>60$ years. Moreover, currently when the lifespan is increasing, usually patients at 65 are without any features of frailty; for this reason, we have decided to have a cut-off point of 70 years.

All patients were carefully followed with median follow-up time for survivors of 12 months (range: 1-21). Overall survival (OS) time was calculated from the date of the start of immunotherapy to the date of the most recent follow-up or death. Progression free survival (PFS) time was calculated from the date of the start of immunotherapy to the date of the most recent follow-up, or disease progression. PFS and OS were estimated using Kaplan-Meier analysis and expressed as median values with corresponding two-sided 95\% confidence intervals (Cls), and the log-rank test was used for bivariate comparisons. The $\chi^{2}$ test was used to investigate the relationship between the categorical parameters. The differences were considered statistically significant if the $p$-values were $<0.05$.

\section{Results}

We analysed 318 patients who received at least one line of immunotherapy due to non-resectable/metastatic melanoma. Median age of the patients was 62 years (range: 17-90), mean age: $60 \pm 14.5$. Eighty-two patients were $\geq 70$ years old (26\%) of which 21 were aged $\geq 80$ years. Median age of patients $\geq 70$ at the time of diagnosis was 77 years (range: 70-90 years). Figure 2 presents the histogram of the population's age. Baseline patient characteristics are shown in Table 1.

Median follow-up was 12 months (range: 1-21 months). In the older patient group ( $\geq 70$ years old) 2 -year OS was $27 \%$ and for patients aged $<70$ it was $28 \%$ ( $p=$ NS). 2-year progression-free survival was $13.7 \%$ in the group $\geq 70$ years old and in patients aged $<70$ it was 13\% ( $p=N S)$. Differences between age groups in median PFS and median OS were not statistically significant (Figures 3 and 4). Disease progression after the first line of immunotherapy was more frequent in patients $<70$ years of age, and similar in both groups after the second line of treatment $(p=0.524)$.

There were no differences in the toxicity of treatment between the group of older and younger patients. Severe AEs (grades 3 and 4) were reported in 82 cases at the $1^{\text {st }}$ line immunotherapy (in $27 \%$ of patients $<70$ years old and in $31 \%$ of patients $\geq 70$ years old). Most often they were liver toxicity, diarrhoea, rash and hypothyroidism. There were no reported deaths resulting from treatment-induced toxicities. Because these data were collected retrospectively, it is possible that toxicities were under-reported, particularly for grades 1 and 2. Survival and safety data are summarized in Table 2.

Co-morbidities were present in $84 \%$ of patients $\geq 70$ years old with the high prevalence of arterial hypertension, cardiovascular disease and/or diabetes without effects of treatment results. The presence of co-morbidities was not associated with an increased risk of immunotherapy $(p=0.790)$.

Patients $\geq 70$ years of age were significantly less likely to have a BRAF mutation ( $p=0.020$ ), which had no influence on overall survival.

\section{Discussion}

The number of cancer patients in the elderly is increasing. Currently, over $50 \%$ of new cancer cases occur 


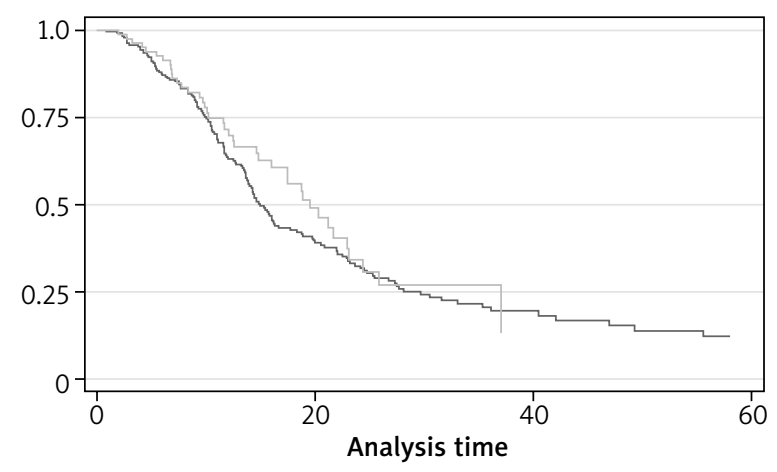

Figure 3. Two-year overall survival according to age (from the start of immunotherapy); $<70$ years old: $28 \%, \geq 70$ years old: $27 \%, p-$ not significant

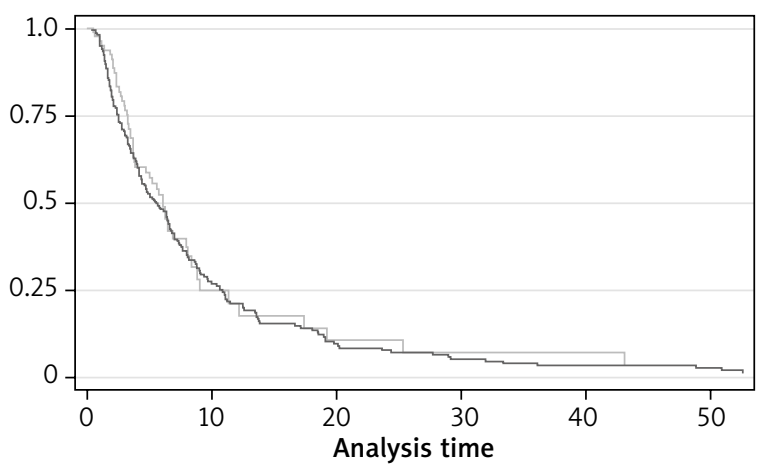

Figure 4. Two-year progression-free survival according to age $<70$ years old: $13.7 \%, \geq 70$ years old: $13 \%, p-$ not significant

Table 2. Survival and safety

\begin{tabular}{|c|c|c|c|}
\hline Parameter & $\begin{array}{l}<70 \text { years old } \\
(236 \text { patients })\end{array}$ & $\begin{array}{l}\geq 70 \text { years old } \\
(82 \text { patients })\end{array}$ & $P$-value \\
\hline Type of immunotherapy as the $1^{\text {st }}$ line of treatment: & 236 & 82 & $\mathrm{~N} / \mathrm{A}$ \\
\hline Anti-PD-1 & 81 & 47 & \\
\hline Ipilimumab & 155 & 35 & \\
\hline Progression on the $1^{\text {st }}$ line of immunotherapy & $200(85 \%)$ & $56(68 \%)$ & 0.002 \\
\hline Toxicity (3/4 grade) ( ${ }^{*}$ assessed in 290 cases) & $61(27 \%)$ & $21(31 \%)$ & 0.525 \\
\hline $\begin{array}{l}\text { Immunotherapy as the } 2^{\text {nd }} \text { line of treatment }- \text { after progression and/or } \\
\text { due to severe toxicity }{ }^{\star} 3 \text { cases): }\end{array}$ & 36 & 10 & $\mathrm{~N} / \mathrm{A}$ \\
\hline Anti-PD-1 & 22 & 7 & \\
\hline Ipilimumab & 14 & 3 & \\
\hline Progression on the $2^{\text {nd }}$ line of immunotherapy & $32(79 \%)$ & $7(70 \%)$ & 0.524 \\
\hline 2-year overall survival & $28 \%$ & $27 \%$ & 0.248 \\
\hline
\end{tabular}

N/A - not applicable.

in people over 65 years of age [17]. The role of immunotherapy in the treatment of cancer also increases. Currently, many clinical trials are conducted with the use of immunotherapy in the treatment of cancer patients. Unfortunately older patients, due to their multiple morbidities and taking many medications, are often excluded from participation in clinical trials with immunotherapy. Only few retrospective analyses and small subgroup analyses in clinical trials are available. For this reason, the use of immunotherapy in elderly patients is a significant problem. It is associated also with a decrease in the efficiency of the immune system in the elderly, which may be the cause of a reduction in the effectiveness of immunotherapy.

Our analysis includes a group of 82 patients $\geq 70$ years treated with immunotherapy and showed that immunotherapy has similar efficacy and toxicity in older and younger patients.

In most studies, the cut-off for age was 65 years [15] but in one of the largest analyses of immunother- apy in the elderly (the Italian study), cut-off for age was 70 years [16]. This study assessed the efficacy and safety of ipilimumab in older patients with pretreated advanced melanoma. A group of 193 patients aged $>70$ years was compared with a group of 662 patients aged $\leq 70$ years. There was no difference in OS, PFS and 1-, 2-year survival rates between patients aged $>70$ years and $\leq 70$ years. There was also no difference in the toxicity of ipilimumab in the younger and older patients.

Similar conclusions were presented in the metaanalysis regarding the comparison of the effectiveness of immunotherapy in older and younger patients [15]. The meta-analysis involved nine clinical trials with anti-CTLA-4 mAb (three with ipilimumab and one with tremelimumab) and five with anti-PD-1 mAb (four with nivolumab and one with pembrolizumab). Five clinical trials concerned treatment of melanoma, four other cancers (renal cancer, lung cancer, prostate cancer). In eight trials, the age of 65 years was accepted as a cut-off point, in one - 70 years. Meta-analysis demonstrated that immuno- 
therapy prolongs OS and PFS in both older and younger patients. Only some studies with nivolumab showed no immunotherapy efficacy in a subgroup of patients over 75 years of age. The authors explained this by the small size of the group, although they did not exclude the influence of weakening of the immune system in patients over 75 years of age. They suggested conducting tests in older patients (> 75 years).

Another retrospective analysis showed similar efficacy of immunotherapy in older and younger patients. This is the analysis of the safety and efficacy of nivolumab in elderly patients with metastatic melanoma [18]. The analysis included 148 melanoma patients, of which 52 (35\%) patients were $>65$ years old.

Other studies describing immunotherapy in older patients concern a small number of patients or are case reports $[19,20]$. Noteworthy is the description of three cases of nonagenarians who were treated with a single agent or a combination of checkpoint inhibitors [19]. Two patients experienced complete or partial responses with acceptable safety profiles, and one other tolerated therapy well although a significant response was not noted.

Our study showed a variable difference in the number of progression after first-line immunotherapy between patients aged $<70$ and $\geq 70$ years. It is probably associated with more frequent use of anti-PD-1 in the first line of immunotherapy in patients $\geq 70$ years of age.

Our study also showed no difference in the effectiveness and safety of immunotherapy in the group of younger and older patients. Also, the analysis of the presence of additional diseases shows no impact on the reduction in the effectiveness of treatment in older patients. Because it is a retrospective analysis, it was not possible to stratify patients by activities of daily living (ADL) and instrumental ADL scales, which would have better characterized the patient population.

In our study, patients $\geq 70$ years of age were significantly less likely to have BRAF mutation ( $p=0.020)$. Age is associated with the changing distribution of BRAFmutant genotype which is also shown by other studies. In a cohort of Australian patients, the frequency of non-V600E genotypes (including V600K) growths with increasing age-decade [21]. In this study, less than 20\% of patients under 50 years with BRAF-mutant melanoma are non-V600E, and $>40 \%$ of patients $\geq 70$ years are non-V600E (70-79). All patients $<30$ years and only $25 \%$ of patients $\geq 70$ years had BRAF-mutant metastatic melanoma. Older patients had a lower prevalence of BRAF mutation and a higher proportion of non-V600E genotypes, predominantly V600K [21]. In our study, there was no correlation between the BRAF mutation and the treatment outcomes of older patients with immunotherapy. However, further research is required as metastatic melanoma in the older patients may be genetically significantly different from melanoma in young patients, which may affect the results of treatment in this group of patients.

\section{Conclusions}

Survival and toxicity in the older patients $(\geq 70$ years) with metastatic melanoma treated with immune checkpoint inhibitors is similar to younger patients ( $<70$ years). The age alone should not exclude the older patients from the use of immune checkpoint inhibitors.

\section{Conflict of interest}

BCS has received for lectures from Novartis, Roche, BMS, MSD, and served as a member of the advisory BMS, IL reaserch projects founded by ROCHE, MSD, BMS. PRhas received for lectures from Novartis, Roche, Pfizer, BMS, Eli Lilly and MSD, and served as a member of the advisory board for Novartis, Merck, Amgen, Blueprint Medicine, Roche, BMS, and MSD. MZ received honoraria from BMS, MDS, Roche, Novartis, Amgen, Pfizer and served as a member of Advisory Board for Novartis, BMS, MSD, Amgen.

Other authors declare no conflict of interest.

\section{References}

1. American Cancer Society: Cancer Facts \& Figures 2017 https://www.cancer.org/research/cancer-facts-statistics/ all-cancer-facts-figures/cancer-facts-figures-2017.html, 10 Jan 2018

2. Balch CM, Soong SJ, Gershenwald JE, et al. Age as a prognostic factor in patients with localized melanoma and regional metastases. Ann Surg Oncol 2013; 20: 3961-8.

3. Haanen JB. Immunotherapy of melanoma. EJC Suppl 2013; 11: 97-105.

4. Postow MA, Callahan MK, Wolchok JD. Immune checkpoint blockade in cancer therapy. J Clin Oncol 2015; 33: 1974-82.

5. Abdul-Karim RM, Cowey CL. Challenging the standard of care in advanced melanoma: focus on pembrolizumab. Cancer Manag Res 2017; 9: 433-42.

6. Fierdman CF, Wolchok JD. Checkpoint inhibition and melanoma: considerations in treating the older adult. J Geriatr Oncol 2017; 8: 237-41.

7. Hodi FS, O'Day SJ, McDermott DF, et al. Improved survival with ipilimumab in patients with metastatic melanoma. N Engl J Med 2010; 363: 711-23.

8. Robert C, Long GV, Brady B, et al. Nivolumab in previously untreated melanoma without BRAF mutation. N Engl J Med 2015; 372: 320-30.

9. Schachter J, Ribas A, Long GV, et al. Pembrolizumab versus ipilimumab for advanced melanoma: final overall survival results of a multicentre, randomised, open-label phase 3 study (KEYNOTE-006). Lancet 2017; 390: 1853-62.

10. Hegde UP, Chakraborty N, Kerr P, Grant-Kels JM. Melanoma in the elderly patient: relevance of the aging immune system. Clin Dermatol 2009; 27: 537-44.

11. Pera A, Campos C, López N, et al. Immunosenescence: implications for response to infection and vaccination in older people. Maturitas 2015; 82: 50-5.

12. Weiss SA, Han J, Darvishian F, et al. Impact of aging on host immune response and survival in melanoma: an analysis of 3 patient cohorts. J Transl Med 2016; 14: 299. 
13. Eisenhauer EA, Therasse P, Bogaerts J, et al. New response evaluation criteria in solid tumours: revised RECIST guideline (version 1.1). Eur J Cancer 2009; 45: 228-47.

14. $\mathrm{NCl}$ Common Terminology Criteria for Adverse Events (CTCAE) v.4.0 data files. (2009) National Cancer Institute, https://evs.nci.nih.gov/ftp1/CTCAE/About.html

15. Nishijima TF, Muss HB, Shachar SS, Moschos SJ. Comparison of efficacy of immune checkpoint inhibitors (ICls) between younger and older patients: a systematic review and metaanalysis. Cancer Treat Rev 2016; 45: 30-7.

16. Chiarion Sileni V, Pigozzo J, Ascierto PA, et al. Efficacy and safety of ipilimumab in elderly patients with pretreated advanced melanoma treated at Italian centres through the expanded access programme. J Exp Clin Cancer Res 2014; 33: 30.

17. Elias R, Morales J, Rehman Y, Khurshid H. Immune checkpoint inhibitors in older adults. Curr Oncol Rep 2016; 18: 47.

18. Freeman M, Weber J. Subset analysis of the safety and efficacy of nivolumab in elderly patients with metastatic melanoma. J Immunother Cancer 2015; 3 (Suppl 2): P133.

19. Johnpulle RA, Conry RM, Sosman JA, et al. Responses to immune checkpoint inhibitors in nonagenarians. Oncoimmunology 2016; 5: e1234572.

20. Sponghini A, Patrucco F, Giorgione R, et al. Complete response to anti-PD-1 nivolumab in massive skin metastasis from melanoma: efficacy and tolerability in an elderly patient. Anticancer Drugs 2017; 28: 808-10.

21. Menzies AM, Haydu LE, Visintin L, et al. Distinguishing clinicopathologic features of patients with V600E and V600K BRAF-mutant metastatic melanoma. Clin Cancer Res 2012; 18: 3242-9. 